\title{
Microstructure and Biocompatibility of Hydroxyapatite Porous Ceramics Designed by a Partial Dissolution-Precipitation Technique with Supersonic Treatment for Bone Regeneration
}

\author{
Toshiyuki Akazawa ${ }^{1}$, Masaru Murata른 \\ Yasuhiko Tabata ${ }^{3}$ and Manabu Ito ${ }^{4}$ \\ ${ }^{1}$ Industrial Technology Research Dept., Hokkaido Research Organization, \\ ${ }^{2}$ School of Dentistry, Health Sciences University of Hokkaido, \\ ${ }^{3}$ Dept. of Biomaterials, Institute for Frontier Medical Sciences, Kyoto University, \\ ${ }^{4}$ Graduate School of Medicine, Hokkaido University,
}

Japan

\section{Introduction}

Hydroxyapatite (HAp: $\left.\mathrm{Ca}_{10}\left(\mathrm{PO}_{4}\right)_{6}(\mathrm{OH})_{2}\right)$ granules and ceramics have been clinically applied for bioactive and substituted materials of hard tissues in dental and medical fields because of excellent biocompatibility and osteoconduction (Oyane, 2010; Yoshikawa, 2009; 2010). In recent years, orthopedists and oral surgeons require the high capability of commercial HAp products for biomaterials, such as improvement of bio-absorption at implanted regions and early incorporation into bio-metabolic system (Artzi et al., 2004; Okuda et al., 2007; 2008). Coping with the required capability of HAp products, it will be necessary to design the biomimetic ceramics that have smooth body-fluid-permeation, appropriate mechanical strength, high surface area, and high affinity for cells.

Satisfying these points, in the previous studies (Akazawa et al., 2005, 2006a; 2006b), we developed functionally graded HAp (designated as fg-HAp) derived from calcined bovine bone; in which grain size and crystallinity of HAp gradually changed from surface regions to bulk parts by utilizing bovine bone calcined at 1073K (b-HAp). HAp porous ceramics made of animal bone can inherit physical and chemical properties of natural resources, such as pore structure and small amounts of metal ions. The fg-HAp ceramics exhibited macropore sizes of $100-800 \mu \mathrm{m}$, porosities of $60-80 \%$, and specific surface areas of $30-40 \mathrm{~m}^{2} \cdot \mathrm{g}^{-1}$.

Bone morphogenetic protein (BMP) accelerates osteoinduction, and it has been added to various scaffold materials due to the growth factor ability to induce bone and cartilage in intramuscular and subcutaneous tissues (Murata et al., 2009a; 2009b; 2010). Interconnected porous HAp can be a superior carrier of recombinant human BMP-2 (rhBMP-2) in ectopic and orthotopic sites. Gradations in the grain size and crystallinity of the HAp plus rhBMP-2addition were used to achieve the desired bio-absorption and osteoinduction characteristics. 
In first vivo-experiment, the fg-HAp and rhBMP-2-loaded fg-HAp (designated as rhBMP$2 /$ fg-HAp) ceramics were implanted into the subcutaneous tissue of the back region in rats (Akazawa et al., 2005; Murata et al., 2007). At 4 weeks after the implantation, for the fg-HAp, body-fluid permeated into bulk regions of HAp through micro-pores, while for the rhBMP2/fg-HAp, fast bio-absorption and osteoinduction were recognized.

In second vivo-experiment, the rhBMP-2/fg-HAp ceramics were implanted into the subcutaneous tissue of the back region in nude mice to look into release characteristics of rhBMP-2 from the ceramics (Tazaki et al., 2006; Kawakami et al., 2007). At 7 days after the implantation, retention percentage of ${ }^{125}$ I-labeled rhBMP-2 was about $60 \%$, suggesting that fg-HAp ceramics are ideal osteoinductive scaffolds.

In third vivo-experiment, the rhBMP-2/HAp ceramics were implanted on the periosteum of biparietal bone in rats (Hino et al., 2008). At 4 weeks after the implantation, degradation and bio-absorption of HAp, bone formation involving HAp fragmented, giant cells around HAp surface, and osteoblasts around new bone were observed. The periosteum played a role of tissue boundary film possible for separation and excision.

If a dissolution-precipitation process for b-HAp porous structure change microstructure of HAp in a very short treatment-time to make up biomimetic fg-HAp ceramics, the process will be expected a design and control-technology of living tissues for bone-regenerative therapy (Akazawa et al., 2007).

Hence, focusing on development and application of the supersonics, ultrasonic echo, ultrasonic knife, ultrasonic microscope, ultrasonic cleaning, ultrasonic disintegration, ultrasonic sterilization and ultrasonic therapy for fracture have been widespread in the medical and dental field (Akazawa et al., 2010a). Supersonic wave more than the frequency of $20 \mathrm{KHz}$ can bring bubble-cavitation and make hot spot. In the hot spot, many chemical reactions will be activated by the formation of radical groups and the locally rising temperature (Rae et al., 2007; Yasuda et al., 2009).

Surface structure design of b-HAp ceramics by a supersonic treatment might easily produce new scaffolds which control the bio-absorption rate and the adsorption ability for proteins or cells. Concerning the commercial HAp products synthesized from reagents, modification of microstructure may be able to improve bio-absorption and tissue-affinity of HAp ceramics by selecting partial dissolution-precipitation conditions (Akazawa et al., 2009c). Provided that a vigorous dissolution-precipitation process for medical HAp products vary microstructure of HAp in a short treatment-time and construct biomimetic HAp ceramics, most of surgeons or medical users will adopt the process as a design and control-technology before the implant-operation for the purpose of advancement of therapeutic grade (Akazawa et al., 2007; 2009b).

In first section, advantage and effectiveness of the fg-HAp ceramics for bone-regeneration together with the supersonic techniques will be introduced and discussed in detail. The spongy b-HAp ceramics using bovine bone were partially dissolved by the two ways, such as stirring for hours and supersonic treatment for minutes. The fg-HAp ceramics originated from bovine or porcine bone were designed by the partial dissolution-precipitation techniques along with the stirring-supersonic treatment. Effects of supersonic treatment- 
condition on the biomimetic microstructure and biocompatibility of fg-HAp ceramics were investigated.

In the second section, the partial dissolution-precipitation techniques along with the supersonic treatment will be applied for surface modification of commercial HAp ceramics. Porous and dense HAp ceramics which have been sold as commercially medical products or research products were easily modified by the partial dissolution-precipitation techniques. Effects of supersonic dissolution-condition on the microstructure of the HAp ceramics were clarified. From in vivo-experiments, histology and histomorphometry of the partiallydissolved and precipitated HAp (designated as PDP-HAp) ceramics were evaluated in contrasting with those of non-treated HAp ceramics.

\section{Surface function design of animal bone-originated HAp ceramics by a partial dissolution-precipitation technique with supersonic treatment}

\subsection{Dissolution characteristics of spongy bone-originated HAp ceramics}

Bovine and porcine bones (Hokkaido Meat Packer Product Industry Ltd. Co.) were used as starting materials and cut to some pieces of the cubic samples. They were boiled to eliminate marrow and most of collagen components from animal bone. They were sequentially calcined at 773-1473K to obtain crystalline HAp (b-HAp) ceramics.

The pulverized cortical b-HAp was completely dissolved into a $\mathrm{HNO}_{3}$ aqueous solution, while the spongy b-HAp was partially dissolved in the $\mathrm{HNO}_{3}$ aqueous solution saturated with respect to apatite by the two different ways, such as stirring at $293 \mathrm{~K}$ and $300 \mathrm{rpm}$ or/and supersonic treatment at $120 \mathrm{~W}$ and 38kHz (Akazawa et al., 2009a; 2009c).

For dissolution characteristics of the ceramics, the dissolution efficiencies were calculated from difference in weight of the ceramics before and after the stirring or supersonic treatment in the $\mathrm{HNO}_{3}$ aqueous solutions.

Figure 1 shows dissolution efficiencies of spongy b-HAp ceramics obtained by the calcination of bovine bone and the supersonic treatment (Akazawa et al., 2009a; 2009c). The dissolution efficiency decreased with increasing calcination temperature of animal bone. This phenomenon would be caused by the decrease in surface area contacting with the $\mathrm{HNO}_{3}$ aqueous solution because b-HAp grains grew and surface area of grain boundary decreased with increasing calcination temperature, although mechanical strength of b-HAp increased by sintering between the particles.

At $1073 \mathrm{~K}$, the dissolution efficiency of b-HAp by the supersonic treatment was significantly higher than that by the stirring. For example, the dissolution efficiency for 5 min-supersonic dissolution was almost same as that for $15 \mathrm{~h}$-stirring dissolution.

Scanning electron microscopic (SEM) and Digital microscopic (DM) photographs of fg-HAp ceramics were taken to observe the morphology and microstructure. Figure 2 shows SEM photographs of the b-HAp ceramics derived from bovine bone and treated by the supersonic dissolution (Akazawa et al., 2009a; 2009c). For the b-HAp calcined at 1073K, grains with less than $1 \mu \mathrm{m}$ and micro-pores were seen, while for the b-HAp calcined at $1473 \mathrm{~K}$, large plate-like grains with 3-8 $\mu \mathrm{m}$ were observed. In both the cases after the 
supersonic treatment, angle-free grains, a lot of micro-pores, and developing micro-cracks were recognized.

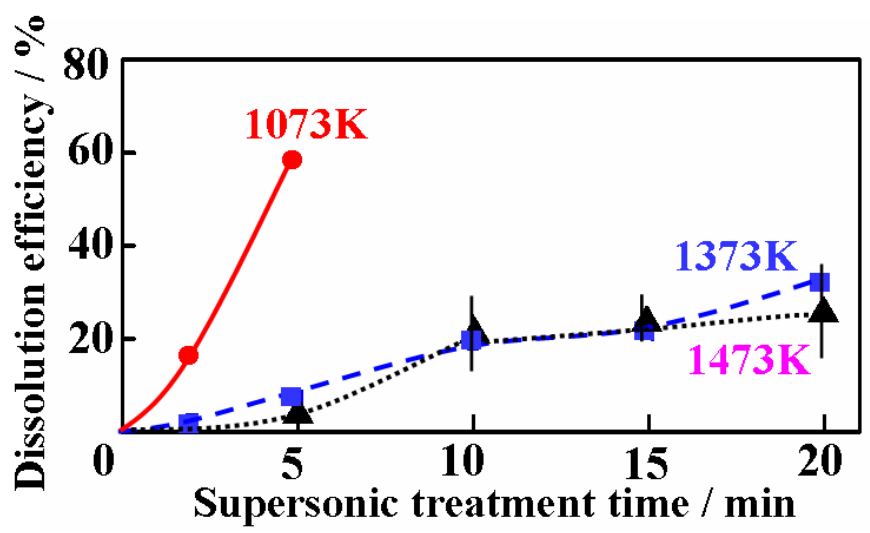

Fig. 1. Dissolution efficiencies of spongy b-HAp ceramics calcined at $1073-1473 \mathrm{~K}$ for $24 \mathrm{~h}$ in air and partially-dissolved by supersonic treatment at $120 \mathrm{~W}$ and $38 \mathrm{kHz}$ in the $\mathrm{HNO}_{3}$ aqueous solutions saturated with respect to apatite.

: b-HAp ceramics calcined at $1073 \mathrm{~K}$.

a: b-HAp ceramics calcined at $1373 \mathrm{~K}$.

$\mathbf{\Delta}$ : b-HAp ceramics calcined at $1473 \mathrm{~K}$.

\subsection{Surface characteristics and microstructure of fg-HAp ceramics derived from animal bone}

Comparing with only the stirring dissolution, the stirring-supersonic dissolution shortened partial dissolution time to one tenth and it made up micro-pores and micro-cracks that are efficient for body fluid permeation. The b-HAp ceramics calcined at $1073 \mathrm{~K}$ derived from bovine or porcine bone were partially dissolved in the $\mathrm{HNO}_{3}$ aqueous solution saturated with respect to apatite by the stirring for $15 \mathrm{~min}$, the supersonic treatment for $2 \mathrm{~min}$, and the stirring for $13 \mathrm{~min}$. The dissolution efficiencies of b-HAp gave $4 \%$ for the first process, $34 \%$ for the second, and $41 \%$ for the third, respectively.

By gradually adding a $\mathrm{NH}_{3}$ aqueous solution at $\mathrm{pH}$ 9-11 and $298 \mathrm{~K}$ into the solution, HAp microcrystals were precipitated on the surfaces and pore-walls of spongy b-HAp. The modified spongy b-HAp was aged for $24 \mathrm{~h}$, washed, and dried at $323 \mathrm{~K}$ to form fg-HAp ceramics (Akazawa et al., 2009c).

The crystalline phase of the samples was identified by Micro-X-ray diffraction (Micro-XRD) using $\mathrm{Cu}$ Ka1 radiation. Micro-XRD patterns of the fg-HAp ceramics showed that in both cases, the degree of crystallinity of HAp single phase gradually distributed better from the pore surface layer to bulk region of the b-HAp body structure. The lattice parameters of HAp phase in the bulk region were $\mathrm{a}=0.942 \mathrm{~nm}$ and $\mathrm{c}=0.688 \mathrm{~nm}$, whose values were in good agreement with those of the JCPDS card (9-432). 


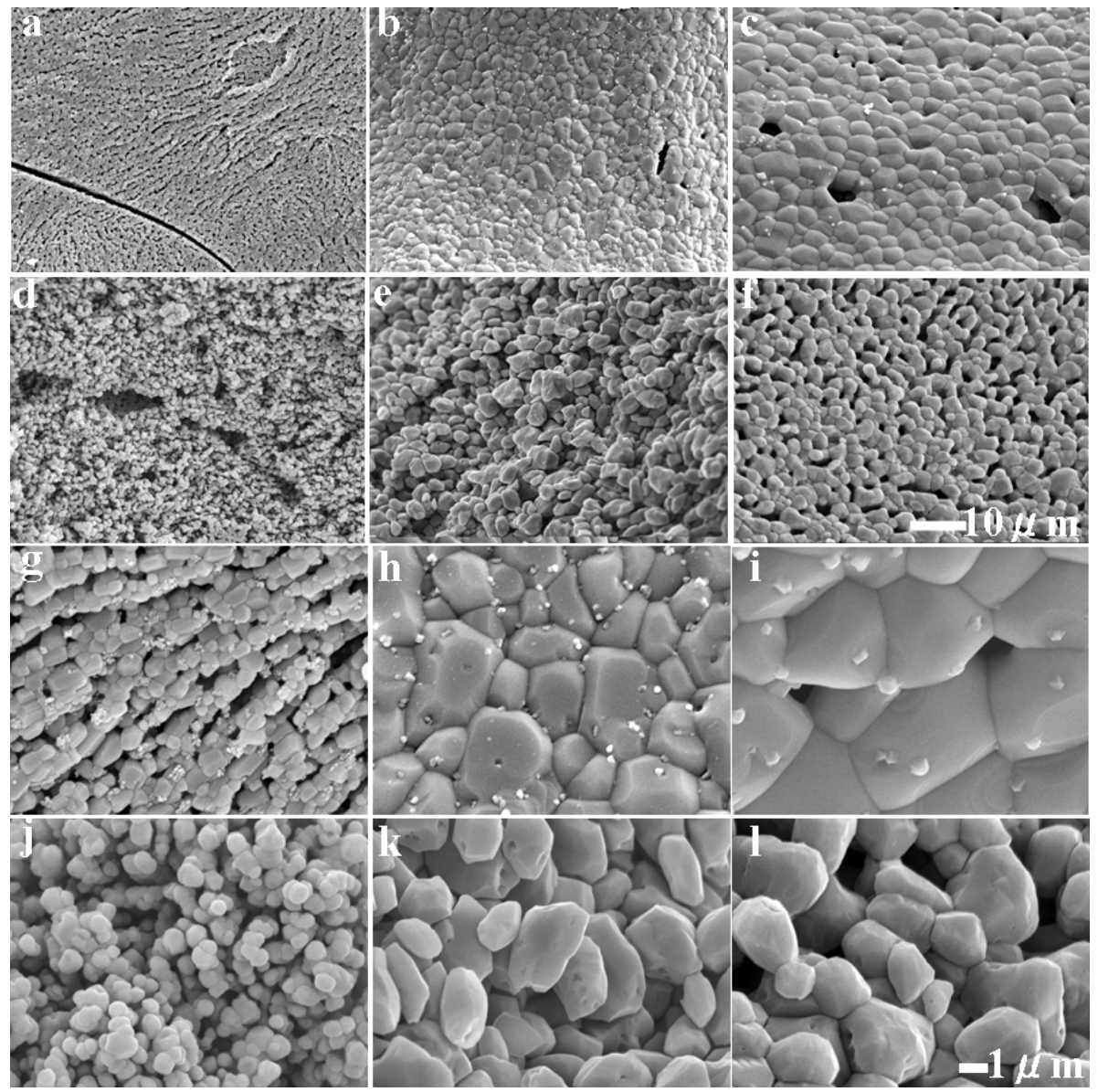

Fig. 2. SEM photographs of spongy b-HAp ceramics using bovine bone calcined at 1073$1473 \mathrm{~K}$ for $24 \mathrm{~h}$ in air and partially-dissolved by supersonic treatment at $120 \mathrm{~W}$ and $38 \mathrm{kHz}$ in the $\mathrm{HNO}_{3}$ aqueous solution saturated with respect to apatite. a: b-HAp ceramics calcined at $1073 \mathrm{~K}$ ( $\times 2000$ magnification). b: b-HAp ceramics calcined at $1273 \mathrm{~K}$ ( $\times 2000$ magnification $)$. c: b-HAp ceramics calcined at $1473 \mathrm{~K}$ ( $\times 2000$ magnification $)$. $\mathrm{d}$ : b-HAp ceramics calcined at $1073 \mathrm{~K}$ and partially-dissolved for $5 \mathrm{~min}$ ( $\times 2000$ magnification). e: b-HAp ceramics calcined at $1273 \mathrm{~K}$ and partially-dissolved for $20 \mathrm{~min}$ ( $\times 2000$ magnification). f: b-HAp ceramics calcined at 1473K and partially-dissolved for $20 \mathrm{~min}$ (×2000 magnification). g: b-HAp ceramics calcined at 1073K ( ×10000 magnification). h: b-HAp ceramics calcined at $1273 \mathrm{~K}(\times 10000$ magnification). i: b-HAp ceramics calcined at $1473 \mathrm{~K}$ ( $\times 10000$ magnification). $\mathrm{j}$ : b-HAp ceramics calcined at $1073 \mathrm{~K}$ and partially-dissolved for $5 \mathrm{~min}$ ( $\times 10000$ magnification). $\mathrm{k}$ : b-HAp ceramics calcined at $1273 \mathrm{~K}$ and partially-dissolved for $20 \mathrm{~min}$ ( $\times 10000$ magnification). l: b-HAp ceramics calcined at 1473K and partially-dissolved for $20 \mathrm{~min}$ (×10000 magnification). 
The composition ratios of $\mathrm{Ca}^{2}{ }^{+}$and $\mathrm{PO}_{4}{ }^{3-}$ ion $(\mathrm{Ca} / \mathrm{P})$ were determined by electron probe microanalysis (EPMA). The quantitative analyses of inorganic components were measured by using inductively coupled plasma (ICP). The fg-HAp was $\mathrm{Ca}^{2+}{ }^{2+}$ deficient HAp with the $(\mathrm{Ca} / \mathrm{P})$ ratios of 1.64-1.66 containing small amounts of $\mathrm{Na}^{+}$and $\mathrm{Mg}^{2+}$ ions.

Figure 3 shows DM and SEM photographs of the fg-HAp ceramics derived from bovine bone (Akazawa et al., 2009a; 2009c). Spherical moss-like grains with $1 \mu \mathrm{m}$ in size, which consisted of about $100 \mathrm{~nm}$ needle-like microcrystals and many micro-pores, were recognized.

Evaluating surface structure, the specific surface areas and pore size distribution were measured from $\mathrm{N}_{2}$-adsorption at $77 \mathrm{~K}$. The porosities of the ceramics were estimated by the water displacement method. The fg-HAp ceramics exhibited porosities of $60-80 \%$, macropore sizes of $100-800 \mu \mathrm{m}$, micro-pores of $10-160 \mathrm{~nm}$, and specific surface areas of $30-50 \mathrm{~m}^{2} \cdot \mathrm{g}^{-1}$.
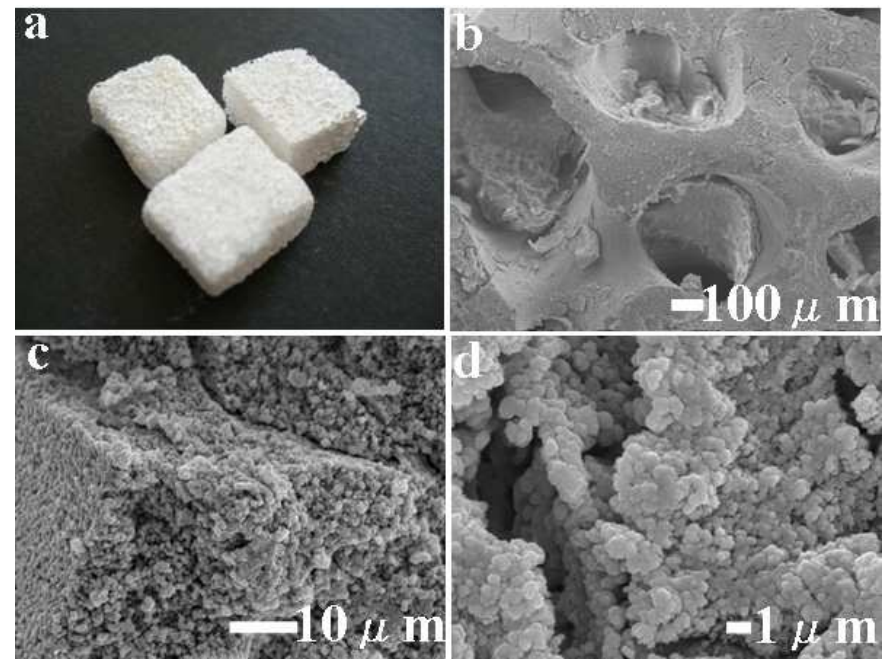

Fig. 3. DM and SEM photographs of fg-HAp ceramics obtained by the partial dissolutionprecipitation of spongy b-HAp calcined at $1073 \mathrm{~K}$ for $24 \mathrm{~h}$ using bovine bone. The dissolution-precipitation involves a stirring for $15 \mathrm{~min}$ in the $\mathrm{HNO}_{3}$ aqueous solution saturated with respect to apatite, a supersonic treatment at $120 \mathrm{~W}$ and $38 \mathrm{kHz}$ for $2 \mathrm{~min}$, a stirring for $13 \mathrm{~min}$, and a precipitation at $298 \mathrm{~K}$ and $\mathrm{pH} 10.5$ for $24 \mathrm{~h}$.

a: DM image of fg-HAp ceramics.

b: SEM image of fg-HAp ceramics (×300 magnification).

c: SEM image of fg-HAp ceramics ( $\times 2000$ magnification).

d: SEM image of fg-HAp ceramics ( $\times 10000$ magnification).

Based on these results above, it is found that the supersonic dissolution-precipitation is an effective process to shorten the partial dissolution time of b-HAp ceramics and design the biomimetic microstructure of fg-HAp ceramics that accelerate degradation-bio-absorption and body fluid permeation of the ceramics in a living body. 


\subsection{Bone-bonding ability of fg-HAp ceramics in a biomimetic environment}

To preliminary evaluate bone-bonding ability, fg-HAp ceramics originated from bovine or porcine bone were soaked at $309.5 \mathrm{~K}$ and $\mathrm{pH} 7.40$ for 4 weeks in a simulated body fluid (SBF) which means the solution where ion concentrations and $\mathrm{pH}$ nearly equal to those in human plasma. Microstructure of the fg-HAp ceramics was shown in Figure 4 (Akazawa et al., 2006c; 2009a).
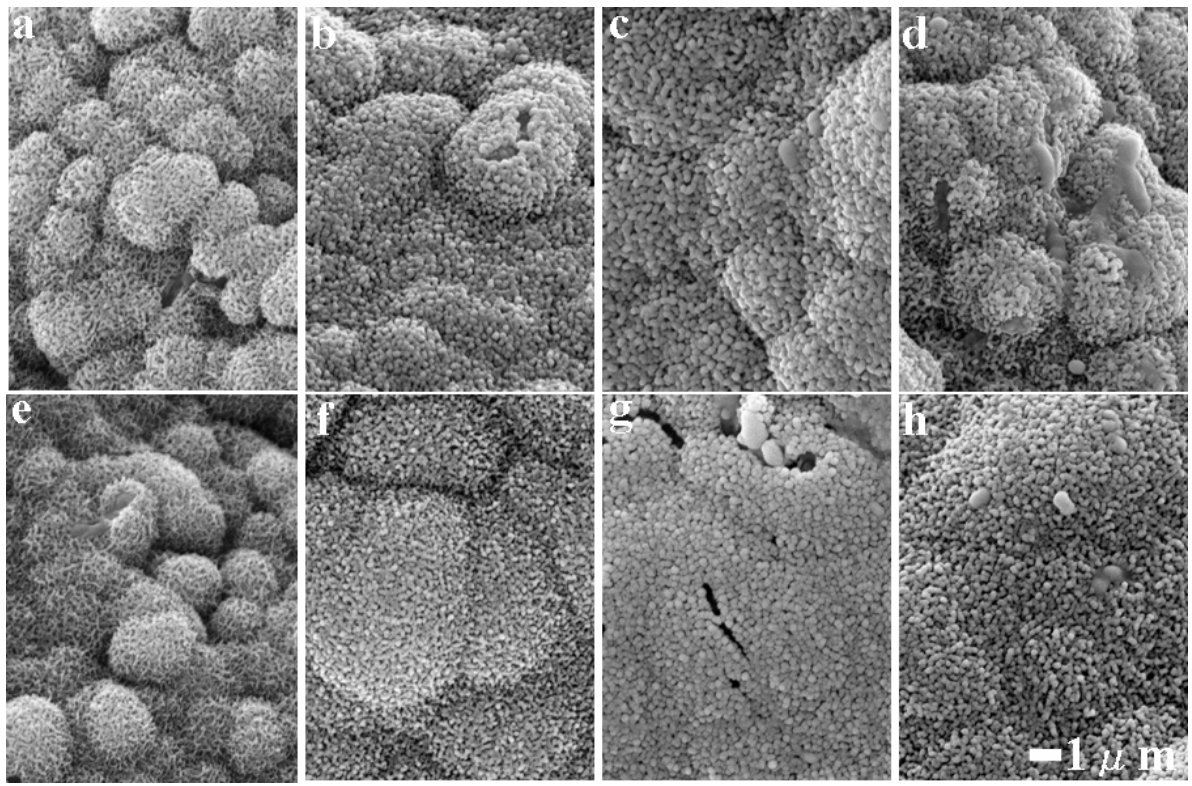

Fig. 4. SEM Photographs of the fg-HAp ceramics soaked at $309.5 \mathrm{~K}$ and $\mathrm{pH} 7.40$ in a SBF solution after the partial dissolution-precipitation with supersonic treatment.

a: bovine bone-originated fg-HAp ceramics at 1 day after the soaking. b: bovine bone-originated fg-HAp ceramics at 3 day after the soaking. c: bovine bone-originated fg-HAp ceramics at 5 day after the soaking $\mathrm{d}$ : bovine bone-originated fg-HAp ceramics at 7 day after the soaking e: porcine bone-originated fg-HAp ceramics at 1 day after the soaking. f: porcine bone-originated fg-HAp ceramics at 3 day after the soaking. $\mathrm{g}$ : porcine bone-originated fg-HAp ceramics at 5 day after the soaking. h: porcine bone-originated fg-HAp ceramics at 7 day after the soaking.

Even at 1 day after the soaking, microstructure of fg-HAp changed from small grains to dense cocoon-like ones by rapid precipitation of HAp microcrystals, suggesting that the fgHAp keeps high bone-bonding ability because ideal biomaterials bond to living bone through an apatite layer formed on the surfaces in a living body. The fg-HAp surfaces will give larger amounts of proteins adsorbed and higher adsorption heats for proteins than the b-HAp surfaces. 


\subsection{Biocompatibility and bio-absorption of fg-HAp ceramics}

The fg-HAp ceramics having sufficient strength for operation were implanted into the subcutaneous tissues of back region in 4-week-old male Wistar rats (Akazawa et al., 2006c; 2009a). At 3 and 8 weeks after the implantation, the sample blocks were explanted, fixed in neutral buffered formalin, decalcified with formic acid, embedded in paraffin, sectioned and stained with hematoxylin and eosin. They were histologically evaluated using an optical microscope.

Figure 5 shows photomicrographs of HE sections at 3 week after the implantation of fg-HAp ceramics (Akazawa et al., 2006c; 2009a). At 3 weeks after the implantation, body fluid well permeated and diffused into bulk regions of HAp through micro-pores of the ceramics. Microcracks in bulk regions of the ceramics formed by the stirring-supersonic treatment would result in auto-degradation and body fluid permeation.
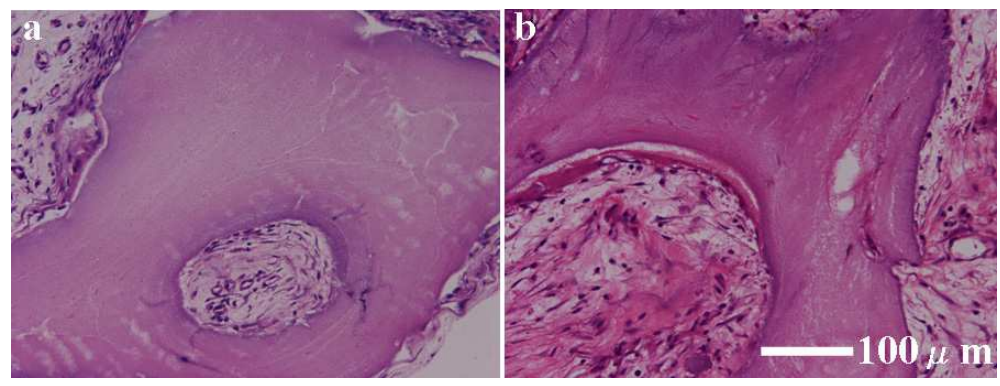

Fig. 5. Photomicrographs of HE sections at 3 week after the implantation of fg-HAp ceramics obtained by the partial dissolution-precipitation of spongy b-HAp calcined at $1073 \mathrm{~K}$ using animal bone.

a: bovine bone-originated fg-HAp ceramics.

b: porcine bone-originated fg-HAp ceramics.

At 8 weeks, the fg-HAp ceramics containing body fluid were degraded from both the surface layer of the ceramics and bulk region, and many giant cells appeared on the surface and inside of the bulk structure of the ceramics. Surface- and bulk-degradations of the fgHAp proceeded, so that a total size of the ceramics dramatically decreased.

\subsection{Conclusions}

Biomimetic microstructure of fg-HAp ceramics was effectively designed using bovine or porcine bone calcined at 1073K (b-HAp) by the partial stirring-supersonic dissolution and precipitation of spongy b-HAp ceramics. The partial dissolution processes of spongy b-HAp ceramics by the stirring at $293 \mathrm{~K}$ for $28 \mathrm{~min}$ in a $\mathrm{HNO}_{3}$ aqueous solution and the supersonic treatment at $120 \mathrm{~W}$ and $38 \mathrm{kHz}$ for 2 min produced convenient micro-pores and micro-cracks for body fluid permeation. The fg-HAp ceramics exhibited macro-pore sizes of $100-800 \mu \mathrm{m}$, porosities of $60-80 \%$ and specific surface areas of $30-50 \mathrm{~m}^{2} \cdot \mathrm{g}^{-1}$.

After the soaking at $309.5 \mathrm{~K}$ and $\mathrm{pH} 7.40$ in a SBF solution, microstructure of fg-HAp changed from small grains to dense cocoon-like ones, suggesting bone-bonding ability and 
biocompatibility of the ceramics. The fg-HAp porous ceramics were implanted into the subcutaneous tissues of back region in rats. At 4 weeks after the implantation, body fluid well permeated from surface into bulk regions of the ceramics and auto-degradation of $\mathrm{fg}$ HAp was recognized.

Based on the results of the soaking tests in a SBF solution and the animal experiments, it was found that the fg-HAp ceramics prepared by the supersonic treatment would be biomimetic materials that indicate fast bio-absorption and excellent biocompatibility for boneregenerative therapy.

\section{Surface function design of commercial HAp ceramics by a partial dissolution-precipitation technique with supersonic treatment}

\subsection{Partial dissolution characteristics of porous HAp products}

Commercial HAp of medical products (HOYA Corporation: APACERAM (G-S-10 granules, AX A-1 ceramics (85\% in porosity), B-4-1010 ceramics (55\% in porosity) (Sakamoto et al., 2007)) were used as starting materials. The G-S-10 granules were completely dissolved in $0.39 \mathrm{~N}-\mathrm{HNO}_{3}$ aqueous solutions to be 1.7 or $3.4 \times 10^{-2} \mathrm{~N}-\mathrm{HNO}_{3}$ aqueous solutions containing

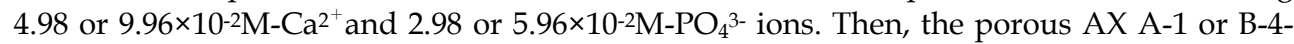
1010 ceramics were cut to $5 \times 5 \times 5 \mathrm{~mm}$-cubic samples and each of the samples was impregnated into the $\mathrm{HNO}_{3}$ aqueous solutions containing $\mathrm{Ca}^{2+}$ and $\mathrm{PO}_{4}{ }^{3-}$ ions saturated with respect to apatite and partially-dissolved by the supersonic treatment at $120 \mathrm{~W}, 38 \mathrm{kHz}, 293-$ 315K and pH 1.0 for 5-75 min (Akazawa et al., 2009b; 2010a). Consequently, degradation of porous ceramics was controlled and frame network of porous ceramics was substantially preserved through the dissolution process.

Figure 6 shows partial dissolution characteristics for the porous HAp products treated by the supersonic treatment (Akazawa et al., 2009b; 2009c; 2010a).

Along with supersonic treatment-time, liquid temperature of the $\mathrm{HNO}_{3}$ aqueous solutions raised from $293 \mathrm{~K}$ to $315 \mathrm{~K}$ because of accumulation of supersonic energy, which implies that the dissolution condition became harder environment.

Dissolution efficiencies of all the HAp products increased with supersonic treatment-time and mechanical strength decreased due to the strong acid etching at $\mathrm{pH}$ 1.0. Higher concentration of the $\mathrm{HNO}_{3}$ aqueous solutions gave higher dissolution efficiency. Dissolution efficiencies for the AX A-1 were much higher than those for the B-4-1010 because the AX A-1 had higher porosity and surface area than the B-4-1010.

In comparison with the stirring dissolution, for the AX A-1 treated by the supersonic dissolution in $1.7 \times 10^{-2} \mathrm{~N}-\mathrm{HNO}_{3}$ aqueous solutions, the dissolution efficiency at the supersonic treatment-time of $15 \mathrm{~min}$ gave $46 \%$, whose value was same as that the stirring time of $6 \mathrm{~h}$. Microstructure of the AX A-1 at the supersonic treatment-time of $30 \mathrm{~min}$ was similar as that at the stirring time at 20h, suggesting an advantage of the supersonic dissolution method. The supersonic dissolution shortened partial dissolution time to about one thirtieth and it made up larger micro-pores and micro-cracks (Akazawa et al., 2009b; 2009c; 2010a). 


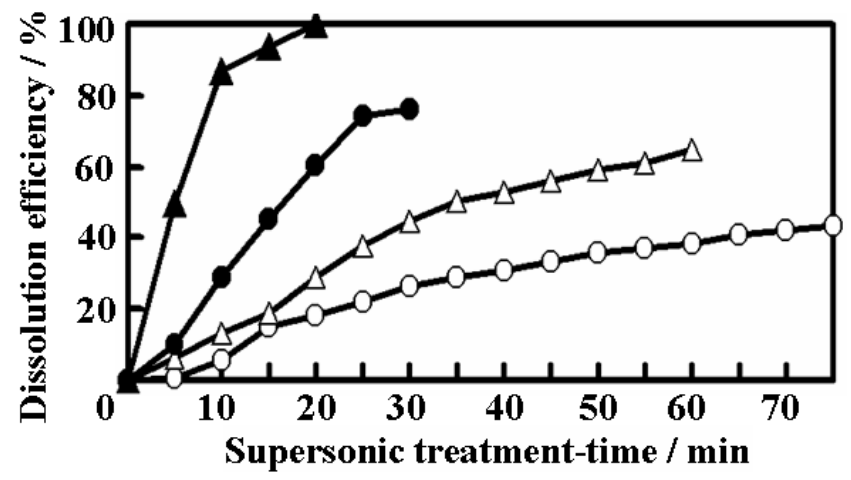

Fig. 6. Partial dissolution characteristics for porous HAp products treated by the supersonic technique at $120 \mathrm{~W}, 38 \mathrm{kHz}, 293-315 \mathrm{~K}$ and $\mathrm{pH} 1.0$ in the $\mathrm{HNO}_{3}$ aqueous solutions containing $\mathrm{Ca}^{2}$ and $\mathrm{PO}_{4}{ }^{3-}$ ions saturated with respect to apatite.

$\boldsymbol{\Delta}$ :AX A-1 ceramics treated in $3.4 \times 10^{-2} \mathrm{~N}-\mathrm{HNO}_{3}$ aqueous solutions.

:AX A-1 ceramics treated in $1.7 \times 10^{-2} \mathrm{~N}-\mathrm{HNO}_{3}$ aqueous solutions.

$\triangle: \mathrm{B}-4-1010$ ceramics treated in $3.4 \times 10^{-2} \mathrm{~N}-\mathrm{HNO}_{3}$ aqueous solutions.

$\bigcirc: \mathrm{B}-4-1010$ ceramics treated in $1.7 \times 10^{-2} \mathrm{~N}-\mathrm{HNO}_{3}$ aqueous solutions.

For the B-4-1010, strong connection between the sintered grains was kept by the supersonic treatment. However, in $3.4 \times 10^{-2} \mathrm{~N}-\mathrm{HNO}_{3}$ aqueous solutions, some craters $100-300 \mu \mathrm{m}$ in diameter, which composed of independent grains, were produced by bubble-cavitation during the supersonic treatment.

Based on these results above, it was suggested that the bioceramics with suitable dissolution efficiency and mechanical strength would be easily designed using commercial HAp products by the supersonic treatment techniques in various acid solutions.

\subsection{Surface characteristics of partially dissolved and precipitated HAp ceramics}

The AX A-1 ceramics with high porosity were selected and partially dissolved in $1.7 \times 10^{-2} \mathrm{~N}-$ $\mathrm{HNO}_{3}$ aqueous solution by the stirring of $300 \mathrm{rpm}$ at $298 \mathrm{~K}$ and $\mathrm{pH} 1.0$ for $30 \mathrm{~min}$ and the subsequently supersonic treatment at $120 \mathrm{~W}, 38 \mathrm{kHz}$ and 293-308K for $25 \mathrm{~min}$. Nano-crystals consisting of calcium phosphate were reprecipitated on the pore-wall surface in the macropores and micro-pores of the ceramics by adding an $\mathrm{NH}_{3}$ aqueous solution. They were aged at $\mathrm{pH} 9-10$ and $298 \mathrm{~K}$ for $24 \mathrm{~h}$ under a bubbling of $\mathrm{N}_{2}$ gas in the solution supersaturated with respect to apatite. The modified HAp was carefully washed with distilled water, and dried at 323-393 K to fabricate the PDP-HAp ceramics (Akazawa et al., 2009b; 2009c; 2010).

The quantitative analyses of $\mathrm{Ca}^{2+}$ and $\mathrm{PO}_{4}{ }^{3-}$ ions in the solutions were conducted using ICP. The concentrations of $\mathrm{Ca}^{2+}$ and $\mathrm{PO}_{4}{ }^{3-}$ ions in the solution after the partial dissolution were $7.93 \times 10^{-2} \mathrm{M}$ and $4.75 \times 10^{-2} \mathrm{M}$, respectively. From Micro-XRD patterns of the PDP-HAp ceramics, single phase of HAp was identified in both the surface layer and bulk region. The $(\mathrm{Ca} / \mathrm{P})$ ratios were 1.64-1.66. The qualitative analysis of carbonate ions was carried out by Fourier transform-infrared spectroscopy and carbonate ions were not detected. 


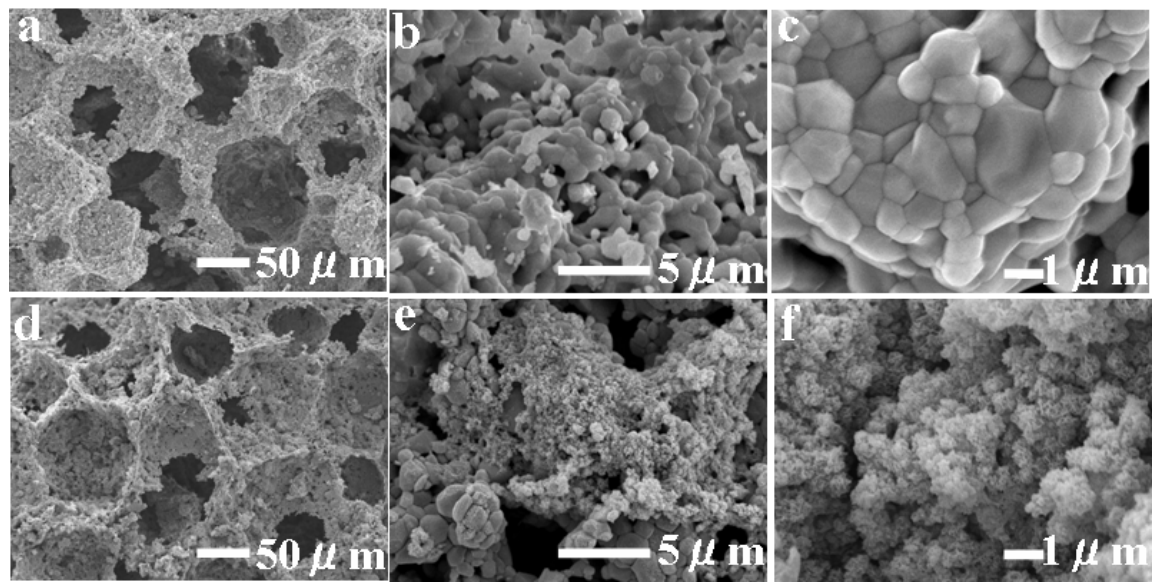

Fig. 7. SEM photographs of HAp ceramics before and after the PDP-treatment involving supersonic dissolution. PDP-HAp ceramics were obtained by the stirring of $300 \mathrm{rpm}$ at $298 \mathrm{~K}$ and $\mathrm{pH} 1.0$ for $30 \mathrm{~min}$ in $1.7 \times 10^{-2} \mathrm{~N}-\mathrm{HNO}_{3}$ aqueous solutions saturated with respect to apatite and the supersonic treatment at $120 \mathrm{~W}, 38 \mathrm{kHz}$ and $293-308 \mathrm{~K}$ for $25 \mathrm{~min}$ and the precipitation at $298 \mathrm{~K}$ and $\mathrm{pH} 9-10$ for $24 \mathrm{~h}$.

a: commercial HAp ceramics (APACERAM AX A-1, HOYA Co.) (×300 magnification). b: commercial HAp ceramics (APACERAM AX A-1, HOYA Co.) (×5000 magnification). c: commercial HAp ceramics (APACERAM AX A-1, HOYA Co.) (×10000 magnification). d: PDP-HAp ceramics ( $\times 300$ magnification). e: PDP-HAp ceramics ( $\times 5000$ magnification). f: PDP-HAp ceramics ( $\times 10000$ magnification).

Figure 7 shows SEM photographs of the PDP-HAp ceramics involving the supersonic treatment (Akazawa et al., 2009b; 2009c; 2010a). Microstructures of the PDP-HAp changed to spherical moss-like grains forming needle-like nano-crystals, micro-pores, and micro-cracks on the grains, which would be efficient for body fluid-permeation and adsorption for plasma-proteins or cells. The PDP-HAp ceramics exhibited macro-pore sizes of 50-200 $\mu \mathrm{m}$, porosities of $80-90 \%$, specific surface areas of $1-2 \mathrm{~m}^{2} \cdot \mathrm{g}^{-1}$.

\subsection{Partial dissolution characteristics of dense HAp products}

To further investigate effects of supersonic dissolution on the microstructure of HAp ceramics, commercial and dense HAp pellets of research products (HOYA Corporation: CELLYARD HAp pellets (13 mm in diameter, no open pore) (Ogawa et al., 2003)) were used as starting materials and treated by the supersonic technique at $120 \mathrm{~W}, 38 \mathrm{kHz}$, and pH 1.0.

For the HAp pellets, many micro-cracks were induced on the almost flat surface by the supersonic treatment for a short time, although the pellets gave low dissolution efficiencies of $1.8-3.8 \%$ in $1.7 \times 10^{-2} \mathrm{~N}-\mathrm{HNO}_{3}$ aqueous solution saturated with respect to apatite.

The microstructure of HAp ceramics before and after the supersonic dissolution-treatment was shown in Figure 8 (Akazawa et al., 2010a). The HAp pellets that were dense HAp ceramics with $95 \%$ in relative density had submicron-grains and closed pores. 
At the supersonic treatment-time of 10-20 min, dense HAp ceramics were significantly etched to form uneven surface and make up many craters 5-10 $\mu \mathrm{m}$ in diameter. Especially, at $20 \mathrm{~min}$, deep and long crinkle-like cracks were formed by accumulation of supersonic energy. Propagation of micro-cracks near the grain boundary in the ceramics would result in inter-and-trans-granular destruction and facilitate bio-degradation in vivo-experiments. The micro-cracks will be very necessary for normal bone-metabolism or bone-remodeling because there are some micro-cracks in various parts and regions of a living bone.
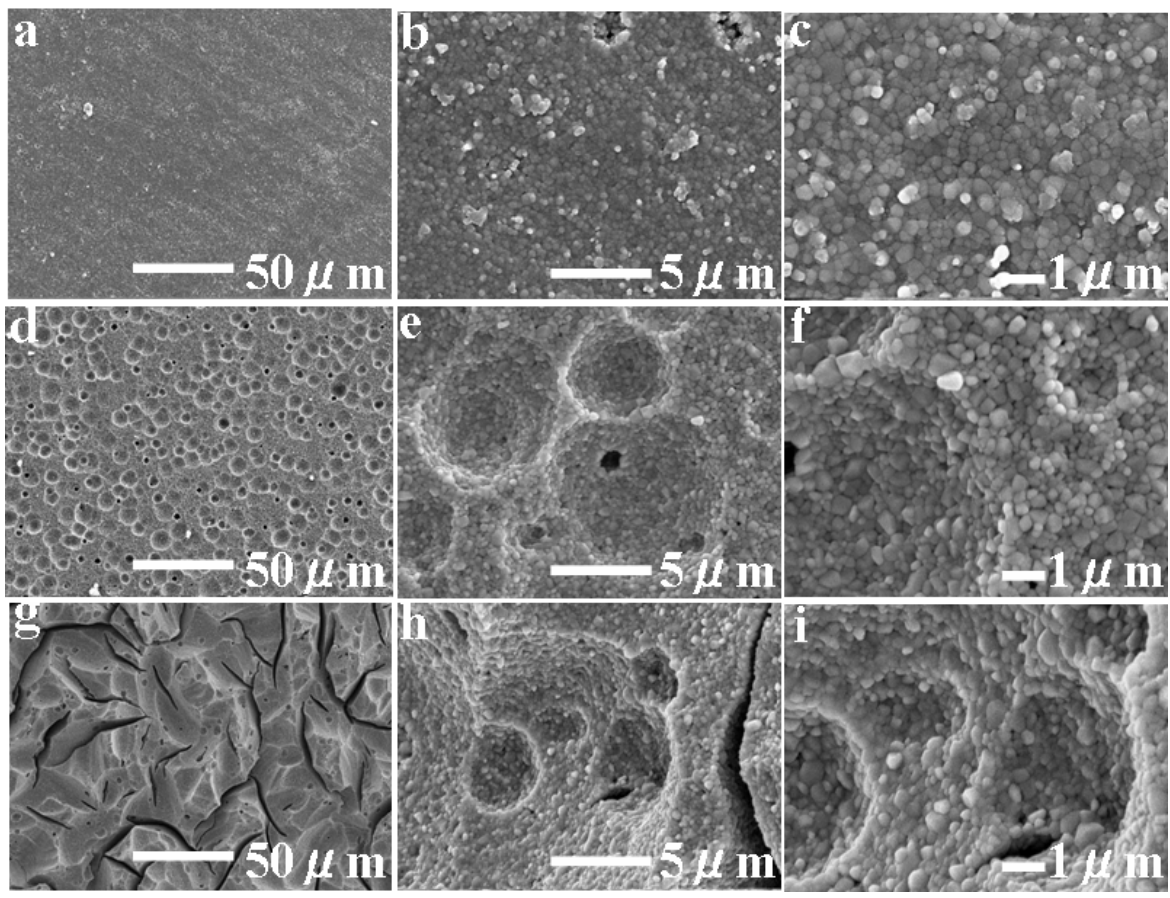

Fig. 8. SEM photographs of dense HAp products treated by the supersonic technique at $120 \mathrm{~W}, 38 \mathrm{kHz}, 293-300 \mathrm{~K}$ and $\mathrm{pH} 1$ in $1.7 \times 10^{-2} \mathrm{~N}-\mathrm{HNO}_{3}$ aqueous solutions saturated with respect to apatite.

a: commercial HAp pellets (CELLYARD, HOYA Co.) (×500 magnification). b: commercial HAp pellets (CELLYARD, HOYA Co.) ( $\times 5000$ magnification). c: commercial HAp pellets (CELLYARD, HOYA Co.) ( $\times 10000$ magnification). $\mathrm{d}$ : commercial HAp pellets treated by the supersonic dissolution for $10 \mathrm{~min}$ ( $\times 500$ magnification). e: commercial HAp pellets treated by the supersonic dissolution for $10 \mathrm{~min}(\times 5000$ magnification).

f: commercial HAp pellets treated by the supersonic dissolution for $10 \mathrm{~min}(\times 10000$ magnification).

g: commercial HAp pellets treated by the supersonic dissolution for $20 \mathrm{~min}$ ( $\times 500$ magnification). h: commercial HAp pellets treated by the supersonic dissolution for $20 \mathrm{~min}$ ( $\times 5000$ magnification). i: commercial HAp pellets treated by the supersonic dissolution for $20 \mathrm{~min}(\times 10000$ magnification). 


\subsection{Bio-absorption and osteoconduction of PDP-HAp ceramics}

Twenty-four adult female of Japanese white rabbits were used and bone defects were bilaterally made at the medial condyle of femur. The HAp products and PDP-HAp ceramics that have sufficient strength for operation were implanted into the bone defects. Eight rabbits were sacrificed at 4,8 , and 16 weeks after the implantation, respectively.

Investigating bio-affinity and biocompatibility of HAp ceramics, both the PDP-HAp and AX A-1 ceramics were implanted into the bone defects in rabbits. Actually, the PDP-HAp ceramics had mechanical strength enough for handling of operation or implantation, in comparison with that of the AX A-1 ceramics.

Micro-computed tomography (Micro-CT) scanning in the axial plane was taken to evaluate the bio-absorption of the implants. Figure 9 shows Micro-CT photographs of different HAp ceramics implanted in the medial condyle of femur in rabbits (Akazawa et al., 2010a; Ding et al., 2010; 2011). At 4-16 weeks after the implantation, the total volume of the PDP-HAp gradually tended to decrease and the shape changed from cubic to angle-free materials, however, those of the AX A-1 were almost similar. Bio-absorbability of the PDP-HAp ceramics will be important for maintaining bone inside implanted ceramics.
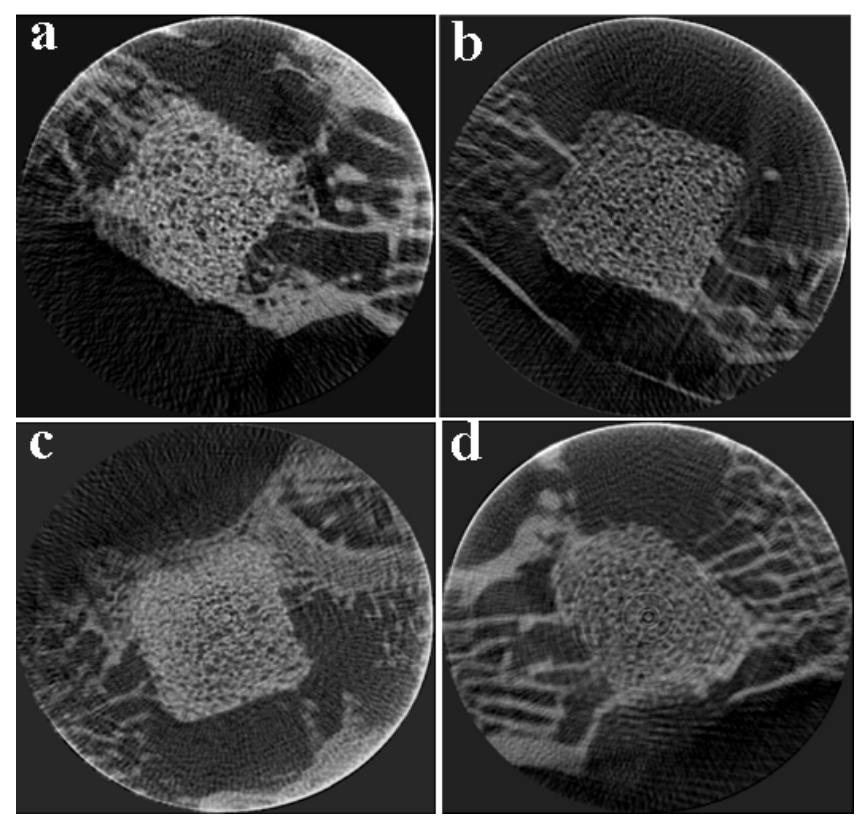

Fig. 9. Micro-CT photographs of different HAp ceramics implanted into the medial condyle of femur in rabbits.

a: AX A-1 ceramics at 4 weeks after the implantation.

b: AX A-1 at 16 weeks after the implantation.

c: PDP-HAp at 4 weeks after the implantation.

d: PDP-HAp at 16 weeks after the implantation. 
The extracted specimens that were not decalcified but stained with toluidine blue $\mathrm{O}$ or tartrate-resistant acid phosphatase (TRAP) were observed by light microscopy.

Figure 10 shows photomicrographs of different HAp ceramics implanted into the medial condyle of femur in rabbits and stained with toluidine blue O (Akazawa et al., 2010a; Ding et al., ; 2011). Although superior osteoconduction for the PDP-HAp and AX A-1 was observed, at 16 weeks, the amounts of bone tissue present for the PDP-HAp were larger than those for the AX A-1, suggesting that the PDP-HAp ceramics induced biological boneremodeling balanced.

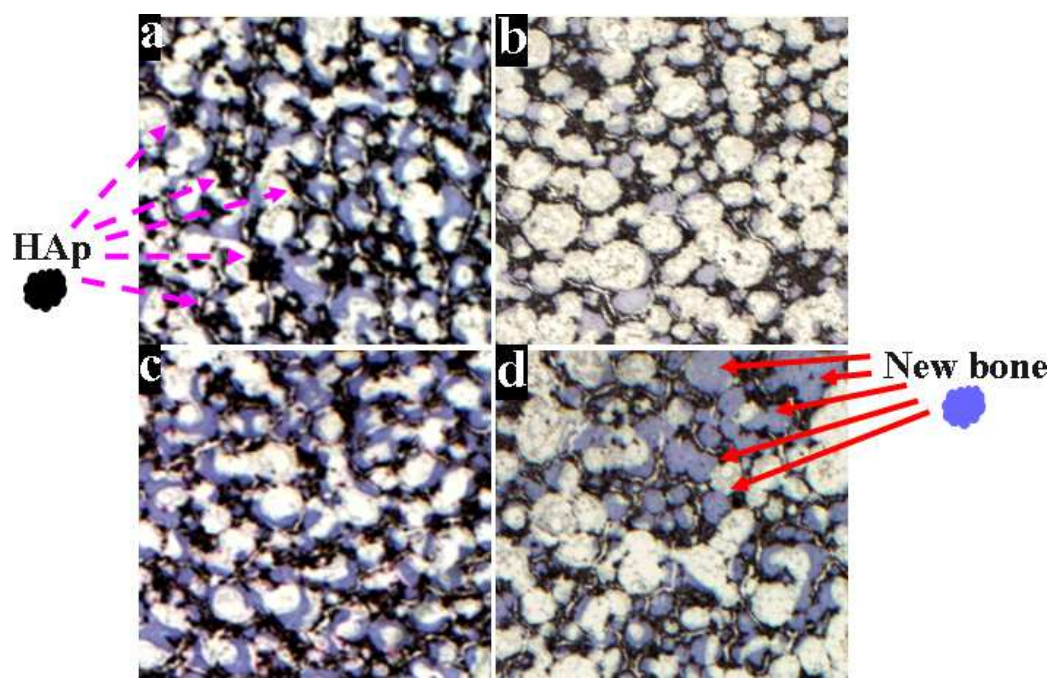

Fig. 10. Photomicrographs of different HAp ceramics implanted into the medial condyle of femur in rabbits and stained with toluidine blue $\mathrm{O}$.

a: AX A-1 ceramics at 4 weeks after the implantation.

b: AX A-1 ceramics at 16 weeks after the implantation.

c: PDP-HAp at 4 weeks after the implantation.

d: PDP-HAp at 16 weeks after the implantation.

At 8 and 16 weeks, other extracted specimens stained with TRAP for both the ceramics indicated multinuclear osteoclasts. For the PDP-HAp, a lot of osteoclasts were directly adsorbed on the surface of HAp and tissue fusion around the ceramics was smoothly advanced. These results can be explained by the body fluid-permeation of PDP-HAp from the surface layers into the bulk regions of the ceramics through the micro-pores or microcracks. The partial dissolution-precipitation involving the supersonic treatment resulted in surface modification of the AX A-1 ceramics to a sort of fg-HAp structure which can stimulate the activity or differentiation for osteoclasts and accelerate the bio-absorption of HAp ceramics. Moreover, the surface layers of the PDP-HAp, such as nano-crystals may disintegrate on solid-liquid interface in vivo.

Accordingly, it was found that the partially supersonic dissolution-precipitation technique enhanced the bio-absorption ability of HAp and would activate the bone-metabolic system. 


\subsection{Conclusions}

Microstructure of commercial porous HAp ceramics drastically modified to the fg-HAp structure by the specific dissolution-precipitation techniques, which involved a stirring of $300 \mathrm{rpm}$ at $298 \mathrm{~K}$ in the $\mathrm{HNO}_{3}$ aqueous solutions containing $\mathrm{Ca}^{2}$ and $\mathrm{PO}_{4}{ }^{3-}$ ions or /and a supersonic treatment at $120 \mathrm{~W}$ and $38 \mathrm{kHz}$ in the same solutions.

The dissolution efficiency of porous HAp products by the supersonic treatment drastically increased with time, depending on the porosity of ceramics and the concentration of $\mathrm{HNO}_{3}$ aqueous solution. For even dense HAp products, enhancement of micro-pores and propagation of micro-cracks were recognized by the supersonic technique at $120 \mathrm{~W}, 38 \mathrm{kHz}$, and $\mathrm{pH} 1.0$ for 10-20 min. Commercial dense HAp pellets were significantly etched to form uneven surface and make up many craters and thunder-like cracks. The partially supersonic dissolution-treatment will be used as a convenient and effective preparation technology for production of bio-absorbable and bioactive ceramics.

After the stirring of $30 \mathrm{~min}$ and the subsequently supersonic treatment of $25 \mathrm{~min}$, HAp nano-crystals with the $(\mathrm{Ca} / \mathrm{P})$ of $1.64-1.66$ were successfully precipitated on the pore wall surface in the macro-pores and micro-pores of the ceramics at $298 \mathrm{~K}$ and pH 9-10.

The PDP-HAp ceramics that gave macro-pore sizes of 50-200 $\mu \mathrm{m}$ and porosities of $85-90 \%$ were implanted into the bone defects at the medial condyle of femur in Japanese white rabbits. At 8 and 16 weeks after the implantation, the PDP-HAp ceramics exhibited more excellent bio-absorption and tissue-affinity than commercial HAp products because of smooth body-fluid-permeation and effective surface nature for cell-adsorption. Superior osteointegration of the PDP-HAp induced biological bone-remodeling balanced.

The PDP-HAp ceramics may be clinically applied for bone tissue-substituted materials with excellent osteointegration when they are completely bio-absorbed in vivo by optimizing the partial dissolution-precipitation conditions..

\section{Biomaterials controlling biological balance and sonochemistry}

Microstructure and chemical nature-control of biomaterials is an inevitable and great challenge for bone-regenerative medicine therapy to increase therapeutic grade and improve quality of lives of patients. In these days, we are focusing on development and application of the bioabsorbable and biomimetic materials synchronized with living tissues and reformed by the partial dissolution-precipitation techniques with supersonic treatment (Akazawa et al. 2010a).

Concerning an ideal implantation cure of biomaterials, it will important for the cell or growth factor-loaded bioceramics and the gene-transferred biomaterials that were coated with a thin HAp layer having protein and DNA to keep donating suitable amounts of physical and chemical stresses for a certain period of time to biological tissues near the implanted region (Akazawa et al. 2010b). This phenomenon would bring high cell-activity and excellent bone-remodelling of the biological tissues. Moreover, balance control of autonomic nervous system and immune system must be necessary for various cells to accept biomaterials as life environment without allograft rejection and continue receiving moderate stress. 
From the viewpoints of creating biomaterials controlling biological balance, the partial dissolution-precipitation method using calcium phosphate aqueous solutions may be efficient for design and preparation of microstructure promoting degradation-bioabsorption and body fluid permeation as a wet synthetic technology which can add biomimetic function into the b-HAp ceramics. Also, commercial bioceramics would be easily modified to reconstruct microstructure and improve bio-absorption and osteoconduction characteristics by optimizing the partial dissolution-precipitation conditions.

Particularly, sonochemistry related to the supersonic dissolution is expected to be applied for bone-regenerative medicine as a simplified design and preparation technology of absorbable and biomimetic bioceramics in the future.

\section{References}

Akazawa, T., Murata, M., Sasaki, T., Tazaki, J., Kobayashi, M., Kanno, T., Matsushima, K.,Itabashi, K., \& Arisue, M. (2005). Bio-absorption and osteoinduction innovation of bone morphogenetic protein-supported functionally graded apatites originated from cattle bone. J. Am. Ceram. Soc., 88.,12., 3545-3548.

Akazawa, T., Murata, M., Arisue, M., Kanno, T., \& Kobayashi, M., (2006a). Japanese Patent, 3718723.

Akazawa, T., Murata, M., Sasaki, T., Tazaki, J., Kobayashi, M., Kanno, T., Matsushima, K., \& Arisue, M. (2006b). Biodegradation and bioabsorption innovation of the functionally graded cattle-bone-originated apatite with blood compatibility. J. Biomed. Mater. Res., 76A., 1., 44-51.

Akazawa, T., Murata, M., Tazaki, J., Nakamura, K., Itabashi, K., Kanno, T., \& Kobayashi, M. (2006c). Surface structure design and characterization of bioabsorbable and functionally graded apatites originated from bovine bone. Key Engineering Materials, 309-311, 1051-1054.

Akazawa, T., Murata, M., Hino, J., Nakamura, K., Tazaki, J., Kikuchi, M., \& Arisue, M. (2007). Materials design and application of demineralized dentin/apatite composite granules derived from human teeth. Archives of BioCeram. Res., 7., 25-28.

Akazawa, T., Murata, M., Tazaki, J., Nakamura, K., Hino, J., Ito, K., Yamamoto, M., Tabata, Y., Takahata, M., \& Ito, M., (2009a). Biomimetic microstructure and biocompatibility of functionally graded hydroxyapatite derived from animal bone by a supersonic dissolution-precipitation method.Bioceramics, 22., 155-158.

Akazawa, T., Murata, M., Hino, J., Tazaki, J.,Ito, K., Nakamura, K., Takahata, M, Abe, Y., Xianjun, D., Itoh, M., Nakajima, T., \& Sakamoto, M. (2009b). Microstructure and bio-absorption characteristics of hydroxyapatite modified by a partially supersonic dissolution-precipitation technique. Archives of BioCeram. Res., 9., 123-126.

Akazawa, T., Nakamura, K., Itabashi,K., Inano, H., Horikawa, H., Takahashi,H., Yoshinari, S., Murata, M., Tazaki, J., Hino, J., Tabata, Y., Yamamoto, M., Hanawa, T., Yamachika, H., Ohmori, T., \&Kikuchi, M. (2009c). Development of bio-absorbable and biomimetic apatite and its application for bone-regenerative therapy. Reports of the Hokkaido Indus. Res. Inst., 308., 47-55.

Akazawa, T., Murata, M., Takahata, M., Xianjun, D., Abe, Y., Nakamura, K., Hino, J., Tazaki, J., Ito, K., Ito, M., Iwasaki, N., Minami, A., Nakajima, T., \& Sakamoto, M. (2010a). Characterization of microstructure and bio-absorption of the hydroxyapatite 
ceramics modified by a partial dissolution-precipitation technique using supersonic treatment. J. Ceram. Soc. Jpn., 118., 6., 535-540.

Akazawa, T. (2010b). Biomaterials controlling biological balance . Journal of Japanese Society for Biomaterials, 28., 85-86.

Artzi, Z., Weinreb, M., Givol, N., Rohrer, MD., Nemcovsky, CE., Prasad, HS., \& Tal, H. (2004). Biomaterial resorption rate and healing site morphology of inorganic bovine bone and beta-tricalcium phosphate in the canine: a 24-month longitudinal histologic study and morphometric analysis. Int. J. Oral Maxillofac. Implants., 19., 3., 357-368.

Ding, X., Takahata, M., Akazawa, T., Abe, Y., Murata, M., Komatsu, M., Iwasaki, N., Ito, M., \& Minami, A. (New Orleans, March 6-9, 2010). Supersonic modification of crystal surface using partial dissolution and precipitation treatment improves bioabsorbability of synthetic hydroxyapatite. Proceeding of 56th Annual Meeting for Orthopaedic Res. Soc.

Ding, X., D., Takahata, M., Akazawa, T., Komatsu, M., Abe, Y., Iwasaki, N., Murata, M., Ito, M., \& Minami, A. (2011). Improved bioabsorbability of synthetic hydroxyapatite through partial dissolution-precipitation of its Surface. J. Mater. Sci.: Mater. Med., DOI 10.1007 s10856- 011-4291-x.

Hino, J., Murata, M., Akazawa, T., Tazaki, J., \& Arisue, M. (2008). Bone induction by biomimetic functionally graded hydroxyapatite with rhBMP-2 on rat skull periosteum. Int. J. Oral Maxillofac. Implants., 21., 327-337.

Kawakami, T., Kuboki, Y., Tanaka, J., Hijikata, S., Akazawa, T., Murata, M., Fujisawa, R., Takita, H., \& Arisue, M. (2007). Regenerative Medicine of Bone and Teeth-with special references to biological principles, problems and their indicators -. J. Hard Tissue Biology, 16., 95-113.

Murata, M., Akazawa, T., Tazaki, J., Ito, K., Sasaki, T., Yamamoto, M., Tabata, Y., \& Arisue, M. (2007). Blood permeability of a novel ceramic scaffold for bone morphogenetic protein-2. J Biomed Mater Res, 81B., 2., 469-476.

Murata, M., Akazawa, T., Tazaki, J., Ito, K., Hino, J., Kamiura, Y., Kumazawa, R., \& Arisue, M. (2009). Human Dentin autograft for bone regeneration - Automatic pulverizing machine and biopsy -. Bioceramics 22, 22., 745-748.

Murata, M., Kawai, T., Kawakami, T., Akazawa, T., Tazaki, J., Ito, K., Kusano, K., \& Arisue, M. (2010a). Human acid-insoluble dentin with BMP-2 accelerates bone induction in subcutaneous and intramuscular tissues. J. Ceram. Soc. Jpn., 118., 438-441.

Murata, M., Akazawa, T., Takahata, M., Ito, M., Tazaki, J., Hino, J., Nakamura, K., Iwasaki, N., Shibata,T., \& Arisue, M. (2010b). Bone induction of human tooth and bone crushed by newly developed automatic mill. J.Ceram. Soc. Jpn., 118., 434-437.

Ogawa, T., Sugo, K., \& Yamamoto, A. (2003). Ceramic hydroxyapatite “CELLYARD” for cell culture. Cell, 35., 474-476.

Okuda, T., Ioku, K., Yonezawa, I., Minagi, H., Kawachi, G., Gonda, Y., Murayama, H., Shibata, Y., Minami, S., Kamihira, S., Kurosawa, H., \& Ikeda, T. (2007). The effect of the microstructure of b-tricalcium phosphate on the metabolism of subsequently formed bone tissue. Biomaterials, 28., 2612-2621.

Okuda, T., Ioku, K., Yonezawa, I., Minagi, H., Gonda, Y., Kawachi, G., Kamitakahara, M., Shibata, Y., Murayama, H., Kurosawa, H., \& Ikeda, T. (2008), The slow resorption with replacement by bone of a hydrothermally synthesized pure calcium-deficient hydroxyapatite. Biomaterials, 29., 2719-2728. 
Oyane, A., (2010). Development of apatite-based composites by a biomimetic process for biomedical appications. J. Ceram. Soc. Jpn., 118., 77-81.

Rae, J., Ashokkumar, M.,Eulaerts, O., Von Sonntag, C., Reisse, J., \& Grieser, F. (2005). Estimation of ultrasound induced cavitation bubble temperatures in aqueous solutions. Ultrason. Sonochem., 12., 325-329.

Sakamoto, M., Nakasu, M., Matsumoto, T., \& Okihara, H. (2007). Development of superporous hydroxyapatite and their examination with a culture of primary rat osteoblasts. J. Biomed. Mater. Res., 82A, 238-242.

Tazaki, J., Akazawa, T., Murata, M., Yamamoto, M., Tabata, Y., Yoshimoto, R., \& Arisue, M. (2006). BMP-2 dose-response and release studies in functionally graded HAp. Key Engineering Mater., 309-311., 965-968.

Yasuda, K. (2009). Decomposition of chemical compounds by ultrasound and development of sono chemical reactor. THE CHEMICAL TIMES, 212., 2-7.

Yoshikawa, H. (2009). Development and clinical application of interconnected porous hydroxyapatite ceramics for bone tissue engineering. J. Jpn. Soc. Biomaterials, 27., 1723.

Yoshikawa, H. (2010). Development and regeneration of bone. J. Jpn. Soc. Biomaterials, 28., 241-247. 


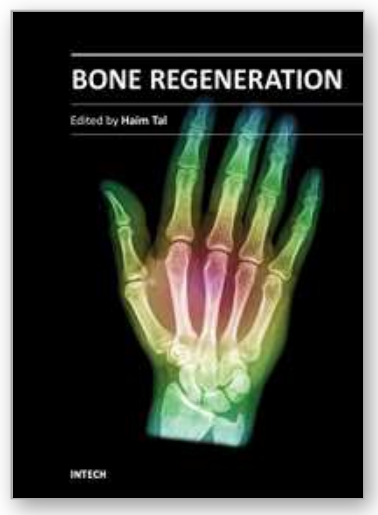

\author{
Bone Regeneration \\ Edited by Prof. Haim Tal
}

ISBN 978-953-51-0487-2

Hard cover, 340 pages

Publisher InTech

Published online 04, April, 2012

Published in print edition April, 2012

Bone is a specialized connective tissue, most prominently characterized by its mineralized organic matrix that imparts the physical properties that allow bone tissue to resist load, to support functional organs, and to protect highly sensitive body parts. Bone loss and bone damage may occur as a result of genetic conditions, infectious diseases, tumours, and trauma. Bone healing and repair, involves integrative activity of native tissues and living cells, and lends itself to the incorporation of naturally derived or biocompatible synthetic scaffolds, aimed at replacing missing or damaged osseous tissues. There are several modalities of bone regeneration including tissue engineering, guided bone regeneration, distraction ontogenesis, and bone grafting. This book concentrates on such procedures that may well be counted among the recent outstanding breakthroughs in bone regenerative therapy.

\title{
How to reference
}

In order to correctly reference this scholarly work, feel free to copy and paste the following:

Toshiyuki Akazawa, Masaru Murata, Yasuhiko Tabata and Manabu Ito (2012). Microstructure and Biocompatibility of Hydroxyapatite Porous Ceramics Designed by a Partial Dissolution-Precipitation Technique with Supersonic Treatment for Bone Regeneration, Bone Regeneration, Prof. Haim Tal (Ed.), ISBN: 978-95351-0487-2, InTech, Available from: http://www.intechopen.com/books/bone-regeneration/biomimeticmicrostructure-and-biocompatibility-of-hydroxyapatite-porous-ceramics-designed-by-a-parti

\section{INTECH}

open science | open minds

\author{
InTech Europe \\ University Campus STeP Ri \\ Slavka Krautzeka 83/A \\ 51000 Rijeka, Croatia \\ Phone: +385 (51) 770447 \\ Fax: +385 (51) 686166 \\ www.intechopen.com
}

\author{
InTech China \\ Unit 405, Office Block, Hotel Equatorial Shanghai \\ No.65, Yan An Road (West), Shanghai, 200040, China \\ 中国上海市延安西路65号上海国际贵都大饭店办公楼405单元 \\ Phone: +86-21-62489820 \\ Fax: $+86-21-62489821$
}


(C) 2012 The Author(s). Licensee IntechOpen. This is an open access article distributed under the terms of the Creative Commons Attribution 3.0 License, which permits unrestricted use, distribution, and reproduction in any medium, provided the original work is properly cited. 